\title{
Anomalous transport in a topological Wannier-Stark ladder
}

\author{
Kun Woo Kim $\odot^{*}$ \\ Center for Theoretical Physics of Complex Systems, Institute for Basic Science (IBS), Daejeon 34126, Republic of Korea \\ and Institut för Theoretische Physik, Universität zu Köln, 50937 Cologne, Germany \\ Alexei Andreanov $₫$ and Sergej Flach \\ Center for Theoretical Physics of Complex Systems, Institute for Basic Science (IBS), Daejeon 34126, Republic of Korea
}

(Received 8 December 2019; revised manuscript received 23 March 2020; accepted 25 March 2020; published 22 April 2020)

\begin{abstract}
A dc (e.g., electric) field with commensurate lattice direction turns a single-particle band structure in $d=3$ dimensions into an infinite set of equally spaced irreducible $(d-1)=2$-dimensional Wannier-Stark (WS) band structures that are spatially localized along the field direction. Particle transport is expected to be suppressed once the WS bands are gapped in energy. The topological character of the irreducible band structure leads to one-dimensional sets of boundary states which fill the energy gaps. As a result, eigenmodes are smoothly connected in energy and space and yield anomalous particle transport throughout the ladder. The number of chiral boundary modes can be tuned by the dc field strength and manifests through the distribution of dissipated energy and spatial motion, and the temperature dependence of angular momentum carried by particles.
\end{abstract}

DOI: 10.1103/PhysRevResearch.2.023067

\section{INTRODUCTION}

Particle transport in quantum mechanical systems is of fundamental interest in condensed matter physics. When exposed to two thermalized reservoirs with different chemical potentials, particles are transferred due to incoherent energy relaxation. For systems with almost perfect conductance such as Dirac semimetals and boundary modes in topological insulators, energy relaxation is mostly confined to the reservoir contacts $[1,2]$. If the chemical potential difference between two reservoirs is increased to values such that the potential drop between neighboring lattice sites is larger than $\hbar / \tau_{\text {inc }}$ where $\tau_{\text {inc }}$ is a characteristic inelastic scattering time, a Wannier-Stark (WS) ladder begins to develop (for a review, see Refs. [3,4]).

The Stark effect is well known in the study of atomic energy splitting by external electric fields. When a strong $\mathrm{dc}$ electric field is applied to a lattice system, its electronic band structure shows a similar splitting. A dc (e.g., electric) field with commensurate lattice direction, e.g., parallel to any lattice vector of finite length, turns a single-particle band structure in $d$ dimensions into an infinite set of equally spaced irreducible $(d-1)$-dimensional WS band structures [5]. With the advance of experimental techniques in both superlattice semiconductors [6] and cold atoms in optical lattices [4,7], photonic lattices [8], and bulk GaAs using the transient bias

\footnotetext{
*kkim@thp.uni-koeln.de

Published by the American Physical Society under the terms of the Creative Commons Attribution 4.0 International license. Further distribution of this work must maintain attribution to the author(s) and the published article's title, journal citation, and DOI.
}

technique [9], the WS ladder spectrum has been well confirmed experimentally. WS systems contain the physics of strong electric fields under nonequilibrium conditions [10,11], strongly localized states in space [12], disorder and correlation effects $[13,14]$, and topological characters associated with multiple bands [15-17] and stay in the focus of current research [18-21].

WS Hamiltonians are closely related to time-periodic Floquet Hamiltonians, $H_{\text {Floq }}(t+T)=H_{\text {Floq }}(t)$. A static external electric field $\vec{F}$ can be gauged into a wave vector: $\vec{k} \rightarrow \vec{k}-$ $\vec{F} t$. With discrete translational symmetry it follows $H(k)=$ $H(k+2 \pi / a)(a$ is a lattice constant; we drop the vector notation for convenience), and the gauged WS Hamiltonian $H(k-$ $F t)$ is Floquet time-periodic with period $T=2 \pi /(F a)$. The first attempt to make use of the Floquet formalism to obtain WS states was reported by Glück et al. [22-24]. The connection between a single-particle $d$-dimensional WS Hamiltonian and a $(d-1)$-dimensional Floquet Hamiltonian was later studied with concrete examples carrying nontrivial topological characters in Bloch bands [16,25]. Note, however, that the WS wave functions live in the whole physical $d$-dimensional space, at variance with Floquet systems where wave functions are confined to a $(d-1)$-dimensional physical space.

Recent research into Floquet physics has focused on constructing topological quantum systems [26-30]. At the same time WS research has focused on computing particle currents under strong electric fields as a way to observe the WS ladder [31-33]. In this work, we present a study of edge particle and thermal transport along the dc electric field direction of a WS ladder with a nontrivial topological character in $d=3$ space dimensions. Below a certain electric field strength, onedimensional (1D) boundary modes are generically connecting energy-spaced WS bands. The boundary mode number is controlled by the electric field strength. Because of coupling 
(a)

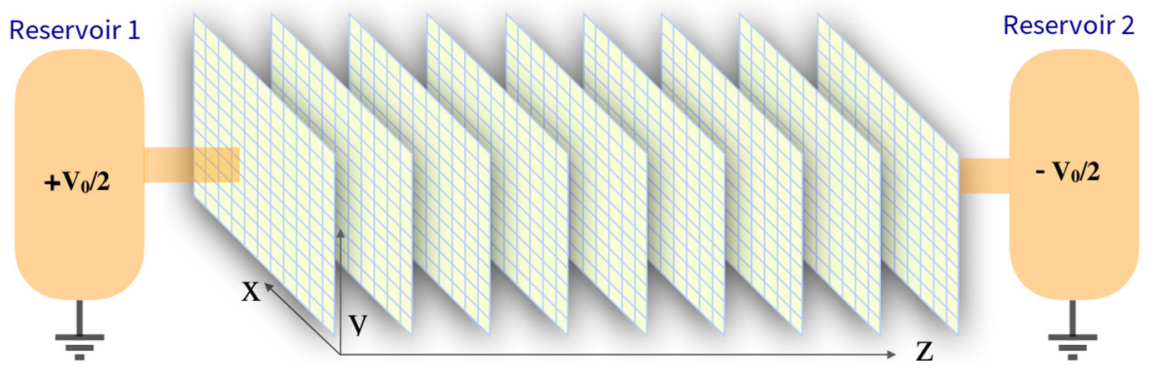

(b)
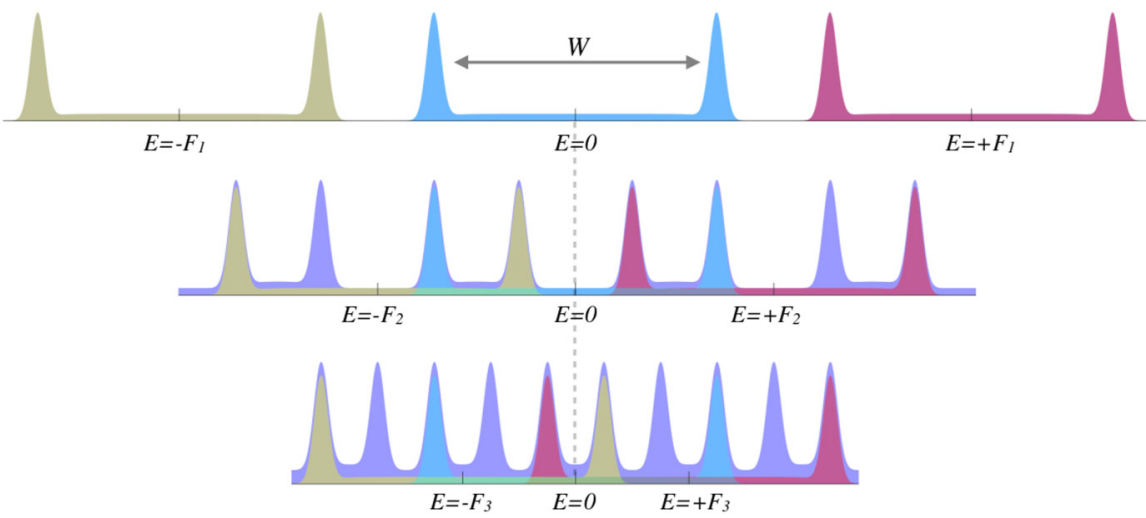

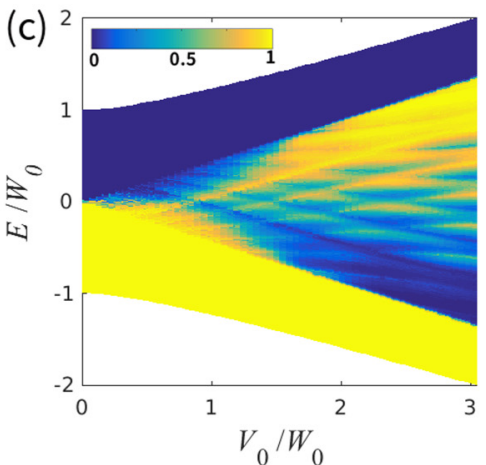

(d)

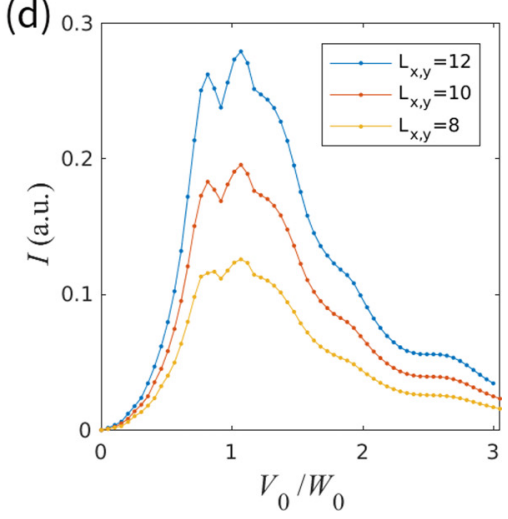

FIG. 1. Sketch of the topological WS ladder. (a) Between two reservoirs with chemical potential difference $V_{0}$, 2D Chern insulators are connected in the $\hat{z}$ direction, along which the system carries a steady particle current. (b) Sketch of band inversion between the WS bands. A strong electric field $F_{1}(>W)$ separates the energy spectrum of each 2D layer, with 1D boundary modes filling the energy gap of a pair of Chern bands. Upon lowering the external field strength $\left(F_{2}<W\right)$, band inversions take place, followed by doubling of the boundary modes at $E= \pm F_{2} / 2$. Further decreasing the electric field $\left(F_{3}<W / 2\right)$, the of boundary modes is tripled at $E=0, \pm F_{3}$. (c) Occupation number of particles as a function of voltage drop $V_{0}$ divided by a bandwidth of the 3D lattice model without bias. (d) Particle current between two reservoirs in steady states with increasing voltage drop at different cross section $L_{x}=L_{y}=8,10,12$ and $L_{z}=9$.

to an incoherent scattering source, particles can propagate along the WS ladder as they relax energy to the bath.

As opposed to in-gap impurity states for which spatially localized modes have random energies and spatial locations, the topological protection of boundary states ensures continuous distribution of eigenmodes in energy space with a finite spatial overlap among neighboring modes. This therefore provides a weak but robust transport of particles across the energy gap regardless of the cutoff energy of heat bath, which otherwise will show transport properties similar to insulators.

\section{TOPOLOGICAL WANNIER-STARK LADDER}

Figure 1 provides a schematic understanding of the energy spectrum of the topological Wannier-Stark ladder (TWSL). A three-dimensional (3D) lattice model is composed of layers of a two-dimensional (2D) Chern insulator with chiral boundary modes circling on the open boundary. When a strong external electric field $F=e V_{0} / L_{z}$ is applied along the $z$ direction, the spectrum of the Chern insulators is identically repeated with every $\Delta E=e a_{z} F$ (lattice constant $a_{z}$ ), forming a set of the WS bands extended in the $x y$ plane while localized in the $z$ direction. The density of states (DoS) within the energy gap
$W$ of two WS bands is filled with chiral boundary modes. In Fig. 1(b) the three sets of WS bands near zero energy are colored to enhance visualization. With decreasing electric field strength, the first band inversion takes place at $E=$ $\pm W / 2$ and is followed by a stepwise increment of the DoS inside the bulk energy gap. With further decreasing of the field strength $(F=1.4 W, F=0.7 W, F=0.4 W)$, energy gaps are flooded with additional sets of boundary modes. Their maximum number is limited by the ratio of the energy gap $W$ and the intrinsic band width and other inelastic scattering sources broadening the spectrum. With the set of chiral boundary modes connecting WS bands, our main question concerns their role in particle transport from one particle reservoir to the other and the related thermal energy emission in the course. Figures 1(c) and 1(d) show the distribution of particle occupancy and the particle current between the two reservoirs in a steady state as the external electric field is tuned, respectively. The details of the calculations will be explained in the following section. The emergence of the WS ladder is shown with increasing voltage drop $V_{0}$ normalized by a bandwidth $W_{0}$ of the 3D lattice model without bias $(F=0)$. The distribution begins to show a dramatic deviation from the Fermi-Dirac one at $V_{0} / W_{0}=1$, and at the same time 
the differential conductance $d I / d V$ turns negative, signifying deviation from the transport of conventional conductors.

\section{MODEL HAMILTONIAN AND PAULI MASTER EQUATION}

To be specific, we employ a model tight-binding Hamiltonian of the Floquet topological insulator on a cubic lattice with two states per site [28]:

$\hat{H}_{\mathrm{WS}}=\sum_{n}[\vec{d} \cdot \vec{\sigma}-F n] c_{n}^{\dagger} c_{n}+\frac{\Delta}{2} \sigma_{3}\left(c_{n+1}^{\dagger} c_{n}+c_{n}^{\dagger} c_{n+1}\right)$,

where $d_{1}=\alpha \sin k_{x}, \quad d_{2}=\alpha \sin k_{y}, \quad d_{3}=\mu-J-2 \beta(2-$ $\left.\left.\cos k_{x}-\cos k_{y}\right)+J \cos k_{x} \cos k_{y}\right)$, and $\vec{\sigma}=\left(\sigma_{1}, \sigma_{2}, \sigma_{3}\right)$ are the Pauli matrices. The lattice spacings are $a_{x}=a_{y}=a_{z}=1$ and $e=1$. The hopping strength between layers $\Delta=10$, and the intralayer hopping strengths $\mu=3, \alpha=4, J=\beta=1.4$, are chosen such that each $2 \mathrm{D}$ WS band carries the Chern number \pm 1 in the large field limit, $F / W_{0} \gg 1$. The index $n$ labels the 2D layers with $H_{2 \mathrm{D}}=\vec{d} \cdot \vec{\sigma}$ in the $x y$ plane, as shown in Fig. 1(a). The lattice translation symmetry is broken in the $z$ direction due to the presence of the electric field $F$, which adds a stepwise increase of potential energy to the $2 \mathrm{D}$ layers. Instead, the model acquires a combined symmetry $\hat{T}_{Z E}$ of discrete translation and energy shift in the $z$ direction $\{n \rightarrow$ $n+1, E \rightarrow E-F\}$, as observed in the energy spectrum of eigenmodes in Fig. 2(a).

We assume a finite extension of the system in the $z$ direction with $-L_{z} / 2 \leqslant z \leqslant L_{z} / 2$ and sizes $L_{x}, L_{y}$ in the $x$ and $y$ directions, respectively. Particles at chemical potential $\mu_{1}=V_{0} / 2$ are released from the reservoir 1 at $z=L_{z} / 2$. For them to reach reservoir 2 with $\mu_{2}=-V_{0} / 2$ at $z=-L_{z} / 2$, a corresponding energy difference $\sim e V_{0}$ must be released or dissipated. We add an incoherent scattering source (for example, phonons) with a well-defined temperature interacting with the fermionic particles in the system. To maintain the integrity of the WS ladder, we assume the coupling strength to be small and the maximum energy carried by one phonon ( $\hbar \omega_{D}$, the Debye frequency) to be smaller than the potential difference between neighboring 2D layers, $F$. We will discuss below the impact of variations $\omega_{D}$ on the transport properties of TWSL. The Pauli master equation, which is valid for the calculation of steady states [34,35], is employed to compute the occupation numbers $f_{n}:=f\left(\epsilon_{n}\right)$ of eigenmodes $n$ :

$$
\begin{aligned}
\frac{d f_{n}}{d t} & =\sum_{m \neq n} P_{n m}, \\
& =\sum_{m \neq n} W_{n m}\left(1-f_{n}\right) f_{m}-W_{m n}\left(1-f_{m}\right) f_{n},
\end{aligned}
$$

where $P_{n m}$ is the scattering rate from eigenmode $m$ to $n$. The scattering strength $W_{n m}$ is determined by the density overlap between two eigenmodes, the temperature of phonon bath, and the phonon DoS (see Appendix A for details).

Figure 1(d) shows the rate of particle number going into reservoir 2 from the TWSL as a function of the electric field strength for three cross-sectional sizes $\left(L_{x} \times L_{y}\right)$ with $L_{z}=9$ in steady states, which is equal to the rate of particle number getting out of reservoir 1 (see Appendix A for the setup

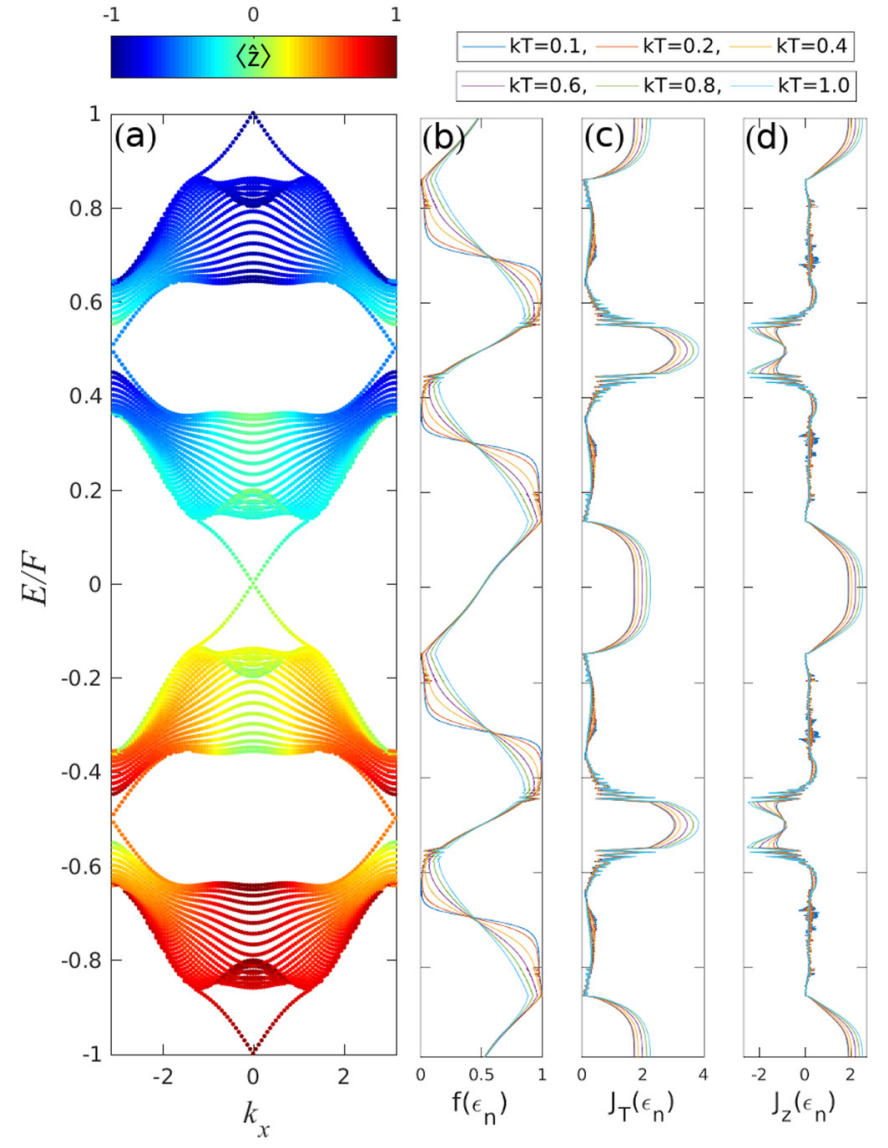

FIG. 2. Energy-momentum dispersion relation and nonequilibrium steady states in the TWSL. (a) $E\left(k_{x}\right)$ at $F=15$. The color code indicates the average position $\hat{z}$ of each eigenmode. (b) Occupation number $f\left(\epsilon_{n}\right)$ in steady states at temperature $k T=0.1-1.0$, filling fraction $v=0.5$. (c) Energy dissipation rate $J_{T}\left(\epsilon_{n}\right)$ associated with each eigenmode $n$. (d) Spatial current $J_{z}\left(\epsilon_{n}\right)$ in the direction of the external field. For the calculation of steady states, we use $N_{x}=N_{y}=$ 22 with open boundary conditions.

of reservoirs). At a small voltage drop $V_{0}=F L_{z}<W_{0}$, the current is increasing with $V_{0}$ as more active transport channels become available. The slope $d I / d V$ is not constant since the DoS depends on energy. On the other hand, when the field strength is further increased, the current decreases with oscillations reflecting the series of energy gap closings in the WS bands. The occurrence of the WS ladder is visible in the map of occupation numbers in Fig. 1(c) through the sequence of occupied and unoccupied energy bands.

\section{NONEQUILIBRIUM STEADY STATES}

Using the model Hamiltonian and the Pauli master equation, in this section we present steady-state results for occupation number $f\left(\epsilon_{n}\right)$, energy dissipation rate $J_{T}\left(\epsilon_{n}\right)$, and spatial currents $J_{z}\left(\epsilon_{n}\right)$ associated with each TWSL eigenmode . These three quantities show distinct behavior in the bulk and chiral boundary modes, signifying their crucial role in the particle transport of the TWSL (see Appendix B for a comparison to in-gap impurity states). 
Figure 2(a) shows the dispersion relation of the TWSL for $F=15$. Here the periodic boundary condition is assumed along the $x$ direction in order to illustrate the chiral boundary mode dispersion in momentum $k_{x}$. With open boundaries at $y= \pm L_{y} / 2$, two boundary modes with opposite chiralities appear in a pair. The color code indicates the expectation value $\langle\hat{z}\rangle$ of the $z$ position of the eigenmodes. While the overall position $\langle\hat{z}\rangle$ decreases with energy the potential energy $V_{\text {pot }}=-F z$ does as well, at energy $E=F / 2$ where boundary modes appear as a result of the band inversion the position $\langle\hat{z}\rangle$ increases with energy. Using the set of eigenmodes of the WS system, we compute particle and energy transport characteristics by solving the Pauli master equation (2). Due to the presence of the symmetry $\hat{T}_{Z E}$ we pick one irreducible eigenmode set, $-F / 2 \leqslant E \leqslant F / 2$, and add periodic boundary conditions in the energy domain. This allows us to compute the steady nonequilibrium state of the system for a region with energy $E$ away from the reservoirs, e.g., $\left|E-V_{0}\right| / F \gg 1$. For the setup of Figs. 2(b)-2(d), we take the number of particles to fill one half of the eigenmodes in the irreducible TWSL band: $v=\sum_{n} f\left(\epsilon_{n}\right) /\left(2 N_{x} N_{y}\right)=0.5$.

Figure 2(b) shows the steady-state occupation numbers of the WS eigenmodes for temperatures $k T=0.1-1$ with $w_{D} / F=0.02$. Within the bulk bands, particles can efficiently relax their energy as there are roughly $\sim 2 L_{x} L_{y}$ scattering channels. On the other side, the boundary modes between two WS bands have only few scattering channels at hand. Therefore, the occupation number is nearly unity at the bottom of each WS band where particles are passing through a bottleneck to enter boundary channels. Then particles are transported down to the top part of the next WS bulk band where the occupation is close to zero. Since the Debye frequency is much smaller than the energy gaps between the WS bands, the interband particle transfer occurs predominantly via boundary modes. Note that with varying temperature the particle occupation of bulk modes follows closely the FermiDirac distribution, $f\left(\epsilon_{n}\right) \simeq 1 /\left(e^{\left(\epsilon_{n}-\mu_{i}\right) / k T}+1\right)$, with the WS band-dependent chemical potential $\mu_{i}$, while the occupancy of boundary modes is essentially independent of the temperature and does not thermalize. As a consequence, certain physical observables such as the angular momentum discussed below show a nontrivial temperature dependence.

Figure 2(c) shows the energy dissipation rate associated with WS eigenmodes:

$$
J_{T}\left(\epsilon_{n}\right)=-\sum_{m \neq n}\left(\epsilon_{n}-\epsilon_{m}\right) P_{n m},
$$

where the scattering rate between eigenmodes is weighted by the amount of released energy. $J_{T}\left(\epsilon_{n}\right)$ is always positive since the scattering events of emitting phonons are more probable than those of absorbing a phonon, $P_{n m}>0$ for $\epsilon_{n}<\epsilon_{m}$ (see Appendix A). The energy dissipation rate per eigenmode $J_{T}\left(\epsilon_{n}\right)$ is largest at $E=0$ and $E= \pm F / 2$ where the particle distribution is far from the equilibrium, while it is suppressed within WS bands, which are well thermalized as reflected through the Fermi-Dirac distribution of their occupancies.

In addition to energy dissipation, the particle transport in the TWSL yields spatial displacements in the direction of the external field. In Fig. 2(d) the spatial current of particles associated with each eigenmode is computed:

$$
J_{z}\left(\epsilon_{n}\right)=\sum_{m \neq n}\left(z_{n}-z_{m}\right) P_{n m},
$$

where $z_{n}=\langle n|\hat{z}| n\rangle$. The spatial current $J_{z}\left(\epsilon_{n}\right)$ is again maximum at zero energy in the chiral boundary modes, while it shows a reversed motion with negative sign at $E= \pm F / 2=$ \pm 7.5 reflecting the presence of inverted WS bands. As is clearly seen in Figs. 2(c)-2(d), both the energy dissipation rate and spatial current show a certain correlation in their magnitudes, which will be further discussed in the section on physical observables.

\section{TOPOLOGICAL PHASE TRANSITION IN TWSL}

As shown in Fig. 1(b), a WS band inversion takes place repeatedly with the decrease of electric field strength $F$. Figure 3(a) shows numerical results for the occupation number $f\left(\epsilon_{n}\right)$ of eigenmode $n$ as a function of energy $E$ and field strength $F$. The eigenenergies as functions of $F$ are plotted with thin solid lines indicating the location of the WS bands and the edge/boundary modes which fill in the energy gap with a relatively larger energy spacing. For three values of the electric field strength $F=12,7$, and 3.5 we show the dispersion relations in Figs. 3(c)-3(e) with the expectation value of $\hat{y}$ in the color coding to emphasize eigenmodes localized near the open surface at $y=-L_{y} / 2$. The above cases demonstrate that the number of $1 \mathrm{D}$ edge/boundary modes localized at the open boundary at $E=0$ and $E= \pm F / 2$ are tuned by the external electric field strength. The boundary modes are connecting fully occupied bulk modes with $f\left(\epsilon_{n}\right) \simeq 1$ (yellow) to almost empty bulk modes $f\left(\epsilon_{n}\right) \simeq 0$ (dark blue) in Fig. 3(a). By releasing energy through scattering off phonons, particles slide down through boundary modes in energy between two WS bands. With a steep gradient of occupation number and only a few channels to scatter into, the rate of thermal energy emission $J_{T}\left(\epsilon_{n}\right)$ is particularly enhanced at $E=0$ and $E= \pm F / 2$ as shown in Fig. 3(b). The two sets of figures show the topological phase transition with external electric field $F$, accompanied by the change of particle occupation in steady states and the thermal energy emission signified by the presence of boundary modes.

\section{EXPERIMENTAL OBSERVABLES}

In the previous section, we showed the comprehensive maps of the occupation number of particles in the topological WS ladder and the map of energy dissipation rate of eigenmodes. As the external electric field $F$ is tuned, the number of boundary modes within the energy gap varies as well as the Chern invariant of the WS bulk bands. While the two quantities, $f\left(\epsilon_{n}\right)$ and $J_{T}\left(\epsilon_{n}\right)$, provide useful microscopic information of the TWSL, eigenenergy-resolved quantities are hardly accessible in experiments. Instead, their energyintegrated statistics can be measured. This section discusses the following physical observables related to the boundary modes in the TWSL: (1) the distribution of dissipated energy, (2) the distribution of spatial displacements of particles in the 
(a)

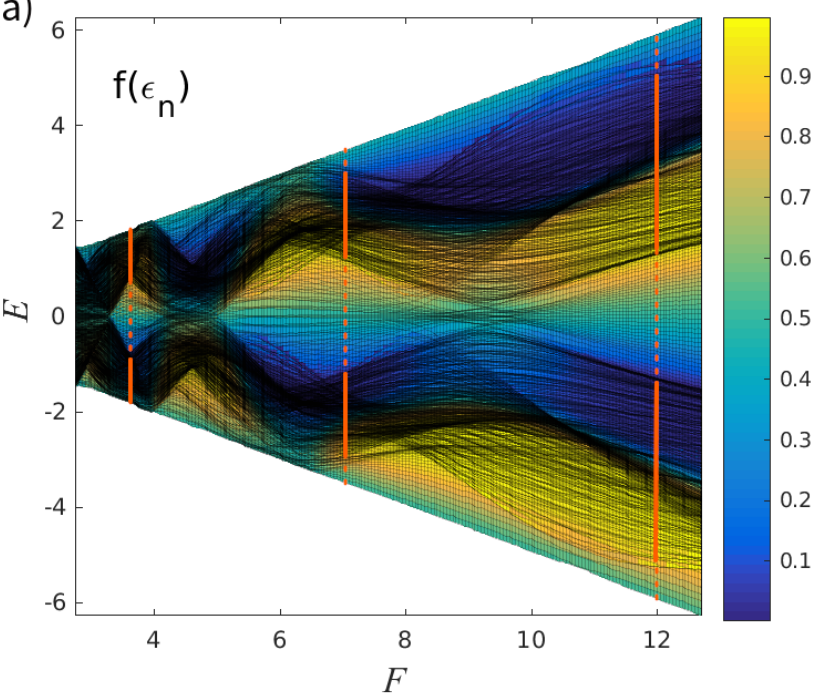

(b)

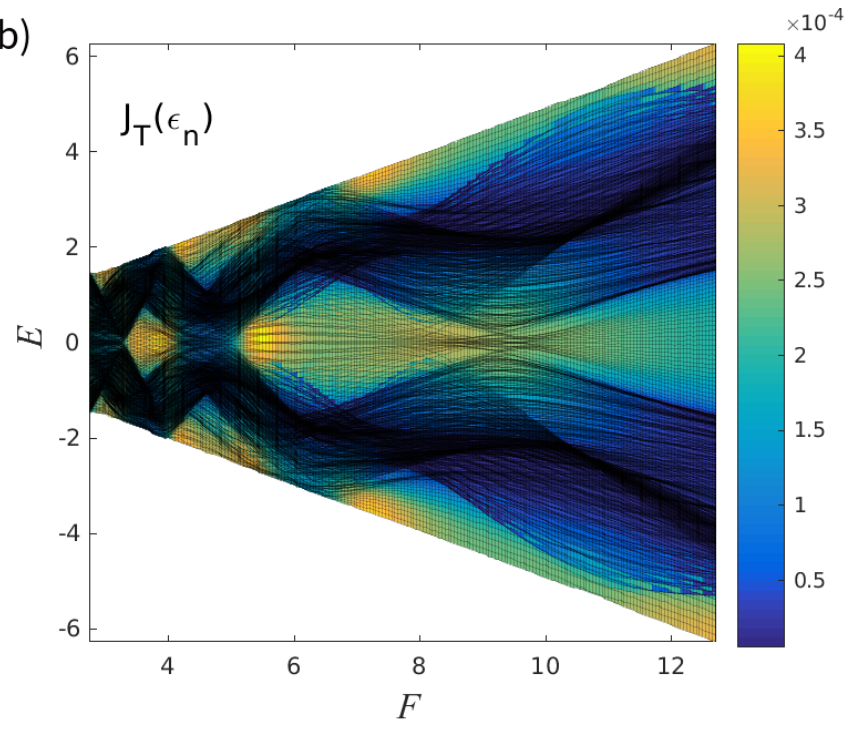

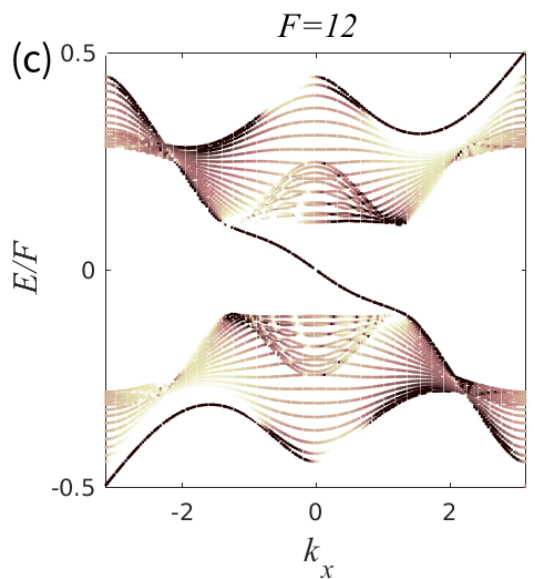
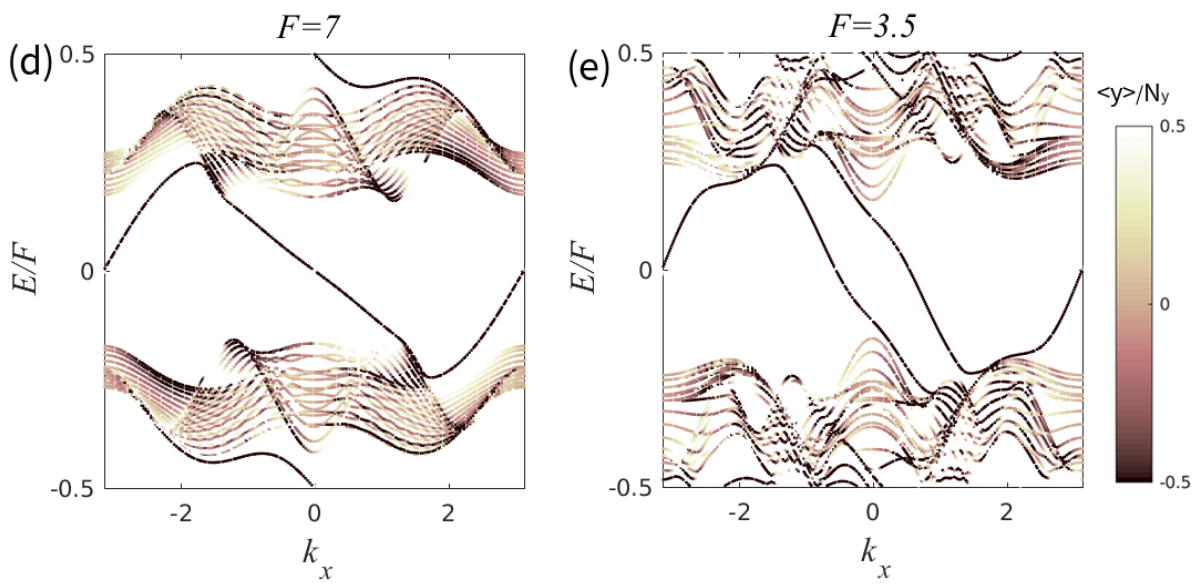

FIG. 3. Energy-resolved occupation numbers and energy dissipation rate. (a) The occupation numbers $f\left(\epsilon_{n}\right)$ of particles in eigenmode $n$, within one irreducible set of WS band in $E \in[-F / 2, F / 2]$ as a function of the external field strength $F$. Thin black lines indicate the trace of eigenenergy in TWSL as a function of $F$. For three values of the external field strength, $F=3.5,7$, and 12 , the energy windows of the WS bulk modes are marked with thick solid vertical lines, and the ones of the chiral boundary modes with dotted lines. (b) Energy dissipation rate $J_{T}\left(\epsilon_{n}\right)$ from scatterings associated with eigenmode $n$. (c-e) Momentum-energy dispersion relation $E\left(k_{x}\right)$ for three representative electric field values $F=12,7,3.5$ with a sizable energy gap. The color coding is chosen to emphasize eigenmodes near the open surface at $y=-L_{y} / 2$ (black).

transport direction, and (3) the sum of angular momentum carried by particles as a function of temperature and filling fraction.

\section{A. Distribution of dissipated energy and spatial displacements}

Figures 4(a) and 4(b) show the distribution of dissipated energy $\Delta E$ and spatial displacements of particles along the $z$ direction $\Delta z$ in the steady state of the TWSL for several Debye frequencies $\omega_{D}=0.3,0.6,0.9,1.2$ :

$$
\begin{aligned}
P(\Delta E) & =\sum_{m, n} P_{n m} \delta\left(\epsilon_{n}-\epsilon_{m}+\Delta E\right), \\
P(\Delta z) & =\sum_{m, n} P_{n m} \delta\left(z_{n}-z_{m}-\Delta z\right),
\end{aligned}
$$

where the sum runs over the eigenmodes of TWSL. To reduce the finite-size effect, $\delta(x) \simeq \pi^{-1} \eta /\left(x^{2}+\eta^{2}\right)$ with $\eta=0.1$ is used. External field strength $F=7$ is chosen without loss of generality. For both plots, the main peaks are located at $\Delta E=\Delta z=0$ and appear due to particles of the bulk modes with occupation close to unity. The distribution is markedly asymmetric reflecting the fact that particles are moving to lower energies and positions $z$. The broad secondary peaks following the main one are caused by interband scattering, whose rate increases with the increase of the Debye frequency $\omega_{D}$. In the presence of boundary modes connecting neighboring WS bands, no matter how small a Debye frequency $\omega_{D}$ is, particles can always find their paths to lower energy, and the dips between the peaks are filled by particle motions via boundary modes.

The particle occupancies $f\left(\epsilon_{n}\right)$ for different $\omega_{D}$ are shown in Fig. 4(c). For small $\omega_{D}$ particles follow the Fermi-Dirac distribution within the WS bands with phonon temperature $k T=0.1$ [see Fig. 3(d) for the energy dispersion relation]. However, with increasing $\omega_{D}$ this quasi-equilibrium is lost, since more and more particles are efficiently 
(a)

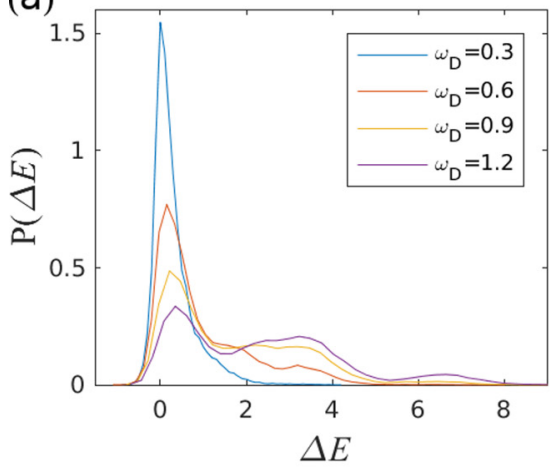

(d)

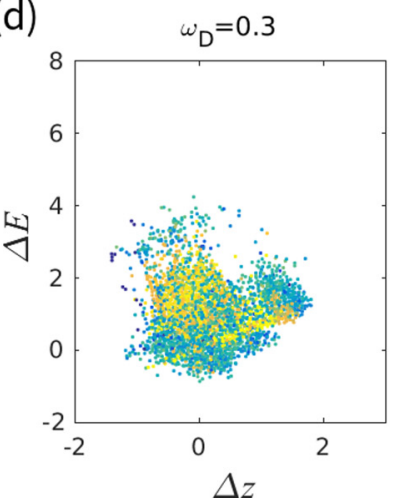

(e) (b)
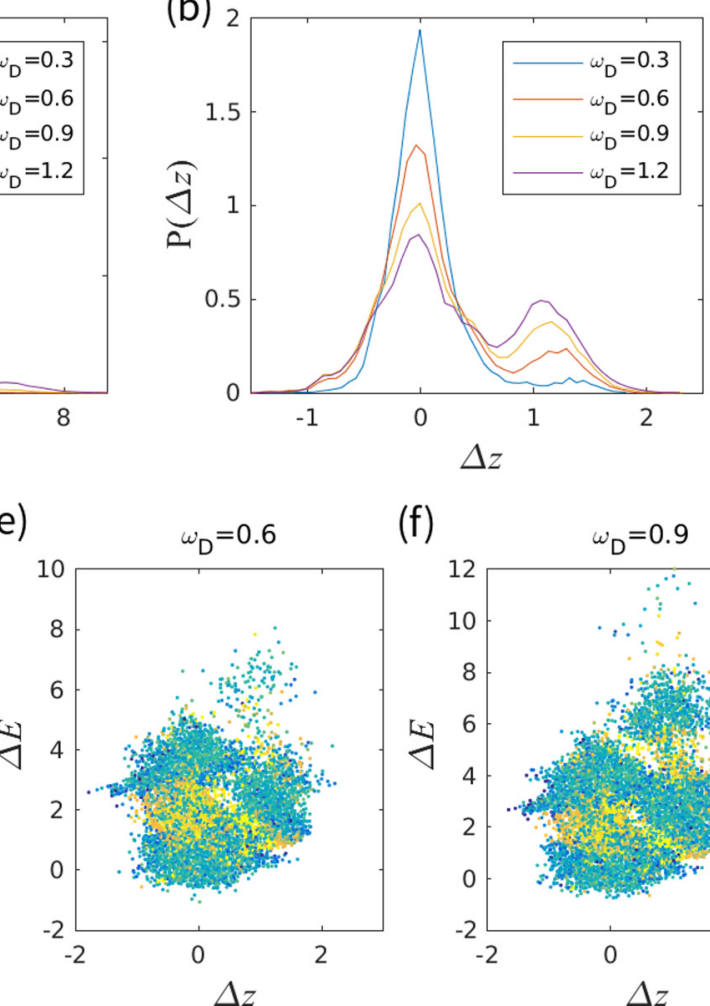

(f) $\quad \omega_{D}=0.9$

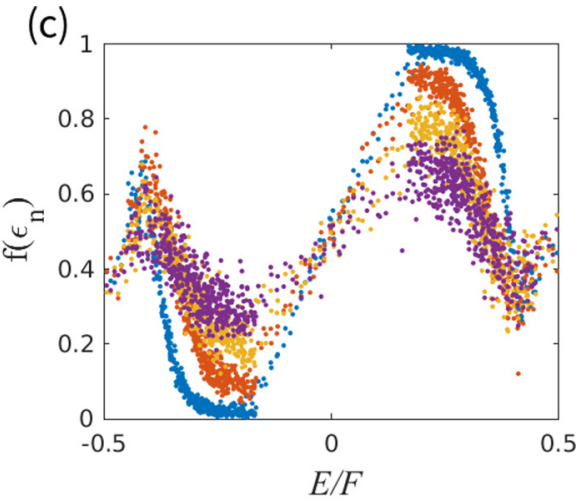

(g)

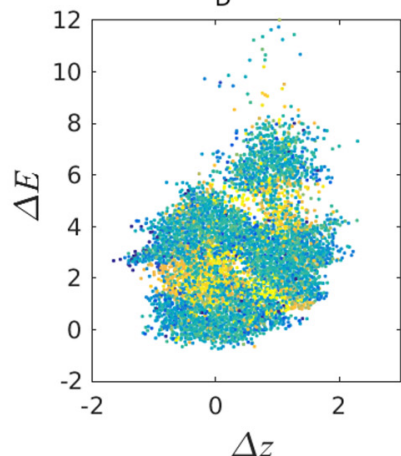

$\langle\hat{\rho}\rangle$

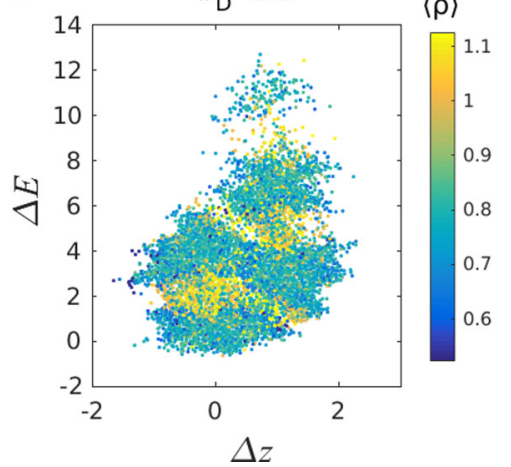

FIG. 4. Dissipated energy and spatial displacements of particles in TWSL. (a-b) Distribution of dissipated energy and spatial displacements of particles along $\hat{z}$ at $F=7, k T=0.1$ for different Debye frequencies: $\omega_{D}=0.3,0.6,0.9,1.2$. (c) Occupation number of particles $f\left(\epsilon_{n}\right)$ for the same set of Debye frequencies (the same color code). (d-g) Correlations of dissipated energy and spatial displacements of particles. Color indicates the expectation value of $\hat{\rho}=\left[\left(2 \hat{x} / L_{x}\right)^{2}+\left(2 \hat{y} / L_{y}\right)^{2}\right]^{1 / 2}$ of eigenmodes associated with scatterings, distinguishing boundary modes from the WS bulk modes: scatterings marked by yellow take place near

scattered to the next WS band via fast direct intraband energy relaxation.

Figures $4(\mathrm{~d})-4(\mathrm{~g})$ show the correlation between dissipated energy $\Delta E$ (vertical axis) and the spatial displacements of particles $\Delta z$ (horizontal axis) for the same set of Debye frequencies, $\omega_{D}=0.3,0.6,0.9,1.2$, for $10^{5}$ scattering events. The color of data points indicates the expectation value of $\hat{\rho}=$ $\left[\left(2 \hat{x} / L_{x}\right)^{2}+\left(2 \hat{y} / L_{y}\right)^{2}\right]^{1 / 2}$ within eigenmodes associated with scatterings, used as a measure distinguishing boundary modes from bulk ones. Interestingly, the parameter space is divided by alternating scattering regions with different colors. If the boundary modes are absent, the regions of bulk scattering modes are disconnected owing to the energy gaps between WS bands. Thus, from the maps of correlations one is able to identify the presence of boundary modes and their role in TWSL.

\section{B. Angular momentum}

Next, we turn our attention to the angular momentum carried by particles in the TWSL. Being localized on the open surface of a 3D lattice, the chiral motion of boundary modes generates a significant angular momentum along the $\hat{z}$ axis. Also the occupation of boundary modes is less sensitive to the temperature compared to the bulk modes, as discussed earlier; see Fig. 2(b). This provides an opportunity to characterize the presence of boundary modes in TWSL from the direct mea- surement of an angular momentum [36] or local magnetic field if particles carry a charge [37]. In the following numerical demonstration, the external field strength $F=15$ is chosen, which corresponds to the setup of Fig. 2.

The angular momentum in the direction of external field is associated with position and velocity operators: $\hat{\mathcal{L}}_{z}=\hat{x} \hat{v}_{y}$ $\hat{y} \hat{v}_{x}$, where $\hat{v}_{j}=i\left[\hat{r}_{j}, \hat{H}\right]$. Thus, the sum of angular momentum of particles in TWSL in a steady state is

$$
\mathcal{L}=\sum_{n} f\left(\epsilon_{n}\right) L_{z}\left(\epsilon_{n}\right),
$$

where $\mathcal{L}_{z}\left(\epsilon_{n}\right)=i\left\langle\psi_{n}|\hat{x} \hat{H} \hat{y}-\hat{y} \hat{H} \hat{x}| \psi_{n}\right\rangle$ is the angular momentum carried by eigenmode $n$. The sum runs over eigenmodes $n$ within an irreducible WS band structure around $E=0$. The occupancies of eigenmode $f\left(\epsilon_{n}\right)$ for different filling fraction $v=\sum_{n} f\left(\epsilon_{n}\right) /\left(2 N_{x} N_{y}\right)=0.1-0.9$ are plotted in Fig. 5(a). With increasing filling fractions, the local chemical potential $\mu_{i}$ of each WS band is increasing to maintain a quasiequilibrium at $k T=0.1$. At the same time, the occupancy of boundary modes stays highly in nonequilibrium with $\frac{d f}{d E}>0$, as they are connecting the two neighboring WS bands.

When the TWSL is completely filled, $f\left(\epsilon_{n}\right)=1$, the sum of angular momentum $\sum_{n} \mathcal{L}_{z}\left(\epsilon_{n}\right)=\operatorname{Tr}\left(\hat{\mathcal{L}}_{z}\right)=0$, because we can always choose a basis, such that $\left|\phi_{n}\right\rangle$ with $\left\langle\phi_{n}\left|\hat{\mathcal{L}}_{z}\right| \phi_{n}\right\rangle=0$ for all $n$. However, for a filling fraction $v<1, \mathcal{L}$ is in general nonzero because the distribution of particle occupation is 
(a)

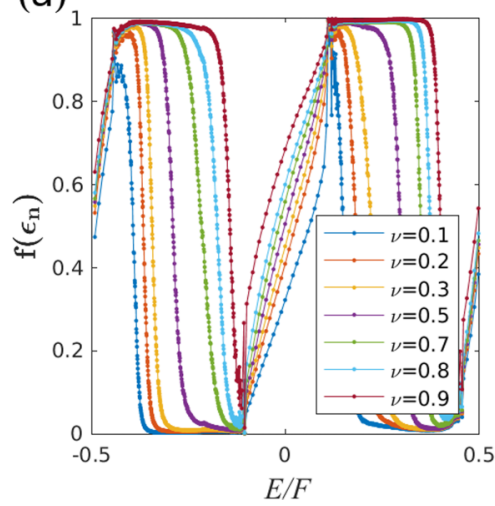

(b)

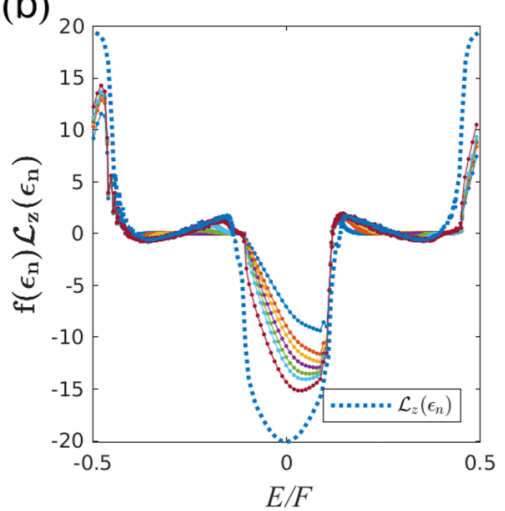

(c)

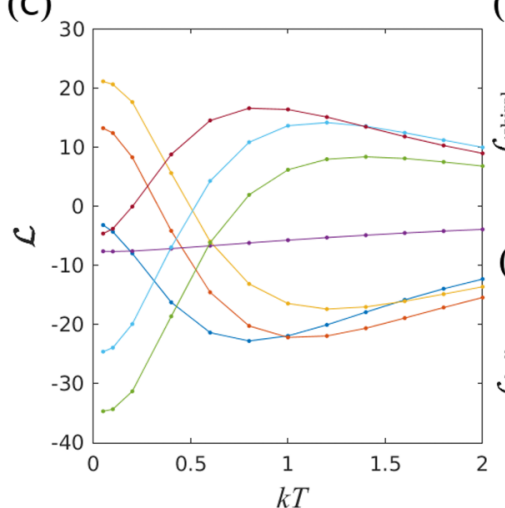

(d)

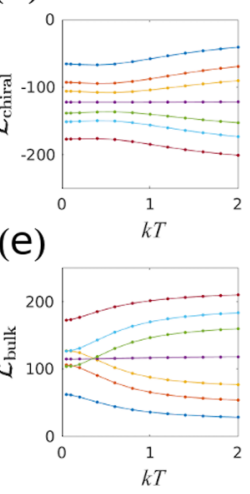

FIG. 5. Angular momentum at different particle fillings and temperature in TWSL. (a) Occupation number of particles $f\left(\epsilon_{n}\right)$ at $F=15$, $k T=0.1$, and filling fraction $v=0.1-0.9$ (legend). (b) Angular momentum carried by particles sitting in eigenmode $n, f\left(\epsilon_{n}\right) \mathcal{L}_{z}\left(\epsilon_{n}\right)$, is plotted in the same set of filling fractions. As a guideline, the angular momentum $\mathcal{L}_{z}\left(\epsilon_{n}\right)$ is plotted with dotted line. (c) The sum of angular momentum $\mathcal{L}=\sum_{n} f\left(\epsilon_{n}\right) \mathcal{L}_{z}\left(\epsilon_{n}\right)$ as a function of temperature for different filling fractions. (d-e) Angular momentum carried by chiral boundary modes $\mathcal{L}_{\text {chiral }}$ and the WS bulk modes $\mathcal{L}_{\text {bulk }}$ are plotted as a function of temperature, $\mathcal{L}=\mathcal{L}_{\text {chiral }}+\mathcal{L}_{\text {bulk }}$.

strikingly different for the WS bulk modes and boundary modes as a function of temperature and filling fraction. The eigenenergy-resolved angular momentum carried by particles, $f\left(\epsilon_{n}\right) \mathcal{L}_{z}\left(\epsilon_{n}\right)$, is shown in Fig. 5(b) for the same set of filling fractions $v=0.1-0.9$. Note that angular momenta of chiral boundary modes around $E=0$ and $E= \pm F / 2$ are distinctively large compared to that of the WS bulk modes, since the former is localized at the open surface with unidirectional group velocity.

Unlike systems in equilibrium where the role of temperature is often reduced to energy broadening and the diminishing of quantum effects, in TWSL the influence of temperature on the angular momentum is dramatic. Figure 5(c) shows the temperature dependence of the sum of angular momentum at different fillings. At half filling $v=0.5, \mathcal{L}$ is nearly insensitive to the change of temperature as a result of the symmetric angular momentum $\mathcal{L}_{z}\left(\epsilon_{n}\right)$ with respect to $E=0$ [see the dotted line in Fig. 5(b)], and the particle-hole symmetric occupation number $f\left(\epsilon_{n}\right) \simeq 1-f\left(-\epsilon_{n}\right)$ [see Figs. 5(a) and 2(b)]. As the filling is tuned away from the half, $\mathcal{L}$ becomes sensitive to temperature and shows abrupt variations. In Figs. 5(d) and 5 (e), $\mathcal{L}$ gets contributions from the boundary modes $\mathcal{L}_{\text {chiral }}$ and bulk modes $\mathcal{L}_{\text {bulk. }}$. Surprisingly, the temperature dependence of $\mathcal{L}_{\text {chiral }}$ and $\mathcal{L}_{\text {bulk }}$ for a given filling fraction marked by the same color shows the opposite behavior, which is the reason for the nontrivial temperature dependence of the sum of angular momentum carried by particles in TWSL.

\section{DISCUSSION}

The Stark effect in a lattice, the WS ladder, was experimentally observed in semiconductor superlattice structures [6] and cold atoms in optical lattices [7]. Despite its continued interest, most experimental and theoretical studies are limited to 1D lattices in a strong field. In this paper, we theoretically study topological phases of a 3D WS ladder, which shares the same topological property with Floquet topological phases in $(2+1)$ dimensions. Moreover, the occupation number of particles in steady states is computed by solving the master equation which allows us to compute transport-related physical observables.

The essential ingredient of our proposal, the topological WS ladder, leads to layers of 2D topological bands and to their coupling in the direction of an applied external field. With intensive interest in topological matter, high-quality 2D topological bands are engineered both in solid state devices [38] and in ultracold atomic setups [39-41]. Thus, the extension to an array of 2D layers as described in Fig. 1(a) is currently within experimental reach. We propose to introduce a constant potential gradient across the $2 \mathrm{D}$ coupled layers to induce the topological WS ladder accompanying the chiral boundary modes in every energy gap between WS bands (see Fig. 3). For this purpose, ultracold atoms in a 3D optical lattice appear to be ideal, where the constant field is generated by gravity in the direction perpendicular to the $2 \mathrm{D}$ layers. The field strength can be tuned by accelerating the optical lattice in the $\hat{z}$ direction, as already demonstrated in previous experiments with 1D structures. Furthermore, the transport of particles can be realized by preparing two particle reservoirs with different chemical potentials [42], for which the motion of particles can be traced and therefore the angular momentum discussed in Sec. VI B can be measured.

\section{ACKNOWLEDGMENTS}

The authors acknowledge financial support from IBS (Project Code No. IBS-R024-D1). K.W.K. is grateful to Carlo Danieli, Prof. J. E. Han, and Hee Chul Park for insightful discussions.

\section{APPENDIX A: SCATTERING MATRIX}

Our calculations assume that the system-bath coupling is sufficiently weak so that its influence on the WS electronic structure is negligible. Scattering between eigenmodes of the TWSL is mediated by incoherent scatterers such as background phonons and photons. The scattering strengths for 

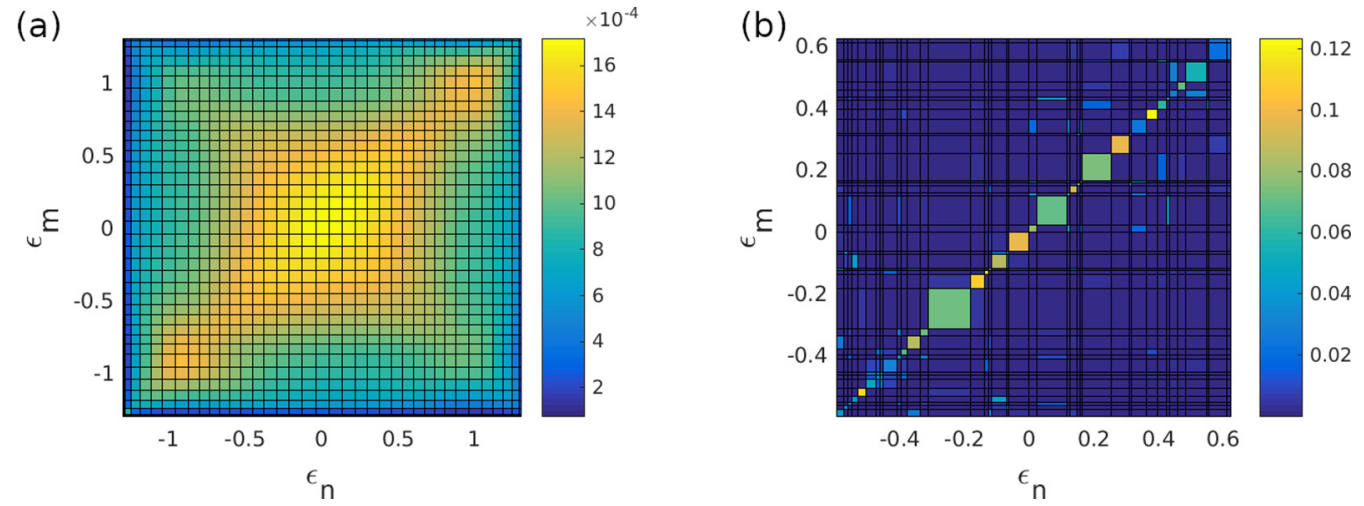

FIG. 6. The overlap of the local DoS between modes $n$ and $m, B_{n m}=\int d^{3} \vec{x} v_{m}(\vec{x}) v_{n}(\vec{x})$, is plotted for eigenmodes of model (i) (a) and model (ii) (b) in the energy gap. See Appendix B for details.

emission and absorption are given by

$$
\begin{aligned}
& W_{n m}=\rho(\omega)\left[1+n_{\omega}\right] \int d^{3} \vec{x} v_{n}(\vec{x}) v_{m}(\vec{x}), \quad\left(\text { for } \epsilon_{n}<\epsilon_{m}\right), \\
& W_{n m}=\rho(\omega)\left[n_{\omega}\right] \int d^{3} \vec{x} v_{n}(\vec{x}) v_{m}(\vec{x}), \quad\left(\text { for } \epsilon_{n}>\epsilon_{m}\right), \quad
\end{aligned}
$$

where phonons absorb/emit the energy difference between two electronic eigenmodes $\omega=\left|\epsilon_{m}-\epsilon_{n}\right|, n_{\omega}=\left(e^{\omega / k T}-\right.$ $1)^{-1}$. The coupling strength between system and bath is $\rho(\omega)=c \omega^{s} e^{-\omega / \omega_{D}}$ where the phonon energy has a Debye frequency cutoff $\omega_{D}$. We take $s=1$ ohmic dissipation. The minimum energy of the phonon is bounded by the system size, $\omega_{b}=c / L_{z}$, and set to $\omega_{b}=0.01$ in our numerics. $v_{n}(\vec{x})=$ $\left|\psi_{n}(\vec{x})\right|^{2}$ is the local DoS of eigenmode $n$ obtained from the diagonalization of the lattice model introduced in Eq. (1).

To compute the particle current across the system in Fig. 1(d), we introduce two thermalized reservoirs with the same temperature as the thermal bath of TWSL, localized at $z= \pm L_{z} / 2$ with chemical potential $\mu= \pm V_{0} / 2$, coupled to eigenmode $n$ in the system with strength $\mathcal{A}_{n}=$ $a \sum_{x, y}\left|\psi_{n}\left(x, y, z= \pm L_{z} / 2\right)\right|^{2}$ at energy $E=\epsilon_{n}$ with a constant $a$. Thus, the tunneling current, for example, to reservoir 2 is computed as

$$
I=\sum_{n} \mathcal{A}_{n}^{\left(z=L_{z} / 2\right)}\left[f\left(\epsilon_{n}\right)-\frac{1}{e^{\left(\epsilon_{n}+V_{0} / 2\right) / k T}+1}\right],
$$

where $f\left(\epsilon_{n}\right)$ is determined from the steady-state solution of the Pauli master equation in (2).

\section{APPENDIX B: TOPOLOGICAL BOUNDARY MODES VS IN-GAP IMPURITY STATES}

The topological protection of boundary modes ensures the presence of eigenmodes continuously distributed in energy space, which in turn leads to a smooth spatial overlap of the local DoS among neighboring modes. Thus, the transport of particles by thermal relaxation remains stable. In spite of the significant reduction of the number of transport channels from the WS band $\left(\sim 2 N_{x} N_{y}\right)$ to the boundary modes $\left[\sim 4\left(N_{x}+\right.\right.$ $\left.N_{y}\right)$ ], the system is not insulating. In contrast, when a bulk energy gap is filled with impurity states, the transport of particles is governed by the scattering of particles from one impurity state to another. Because impurity states are spatially localized in the full 3D space, the scattering strength is strongly reduced compared to that of topological boundary modes. Even worse, the scattering strength is exponentially decaying as a function of energy difference between two modes. Thus, the transport of particles through the energy gap filled with in-gap impurity states is similar to an insulating phase.

In this Appendix we support the above argument by calculating the overlap of the local DoS between mode $n$ and $m$ :

$$
B_{n m}=\int d^{3} \vec{x}\left|\psi_{n}(\vec{x})\right|^{2}\left|\psi_{m}(\vec{x})\right|^{2},
$$

which is a quantity directly related to the scattering strength in Eq. (A1). Note that when $n=m$, the above expression becomes the inverse participation ratio, which is a measure of spatial localization of eigenmodes. In the following we compute $B_{n m}$ for two models: (i) The model introduced in (1) with $F=12$ [see Figs. 3(a) and 3(c) for the related energy spectrum], and (ii) a model with the same set of microscopic parameters $(\mu, \alpha, \beta, J, \beta, F)$ of the clean Hamiltonian but with periodic boundary conditions in the $x$ and $y$ directions so that edge/boundary modes are absent. In addition, for the second model we add an onsite impurity potential:

$$
H_{\mathrm{imp}}=\sum_{n \in X}\left(V_{n} \sigma_{0}\right) c_{n}^{\dagger} c_{n},
$$

where the potential $V_{n}$ is added to a randomly chosen set of lattice sites $X=\left\{n_{1}, n_{2}, \ldots, n_{M}\right\}$ where $M=2 N_{z}\left(N_{x}+N_{y}\right)$, such as to maintain the same number of topological boundary modes as in model (i). The impurity potential is uniformly distributed between $[-2 \alpha, 2 \alpha]$, where $\alpha=4$ is the hopping strength in the $x y$ plane. In this way, in-gap impurity states are introduced between the WS bands.

Figures 6(a) and 6(b) show $B_{n m}$ for models (i) and (ii), respectively, for 40 eigenmodes near zero energy. First, the energy spacings (width between parallel lines) among neighboring topological boundary modes are remarkably uniform due to the level repulsion, while those of impurity modes are random as they are spatially localized in 3D space. Second, it follows that the overlap of the local DoS, $B_{n m}$, of model (i) is the largest for the nearest neighbors, $m=n+1$, and the value is roughly constant regardless of their eigenenergies. This provides advantages for the transport of particles through the topologically protected boundary modes, as the scattering 
strength is exponentially decaying with energy difference, $\omega=\epsilon_{m}-\epsilon_{n}$. In contrast, $B_{n m}$ of model (ii) between impurity modes does not show a conceivable correlation, indicating that the transport of particle through the impurity states is much less efficient.
Last, particles in topological boundary modes in model (i) carry an angular momentum which linearly scales with the system size $L_{x, y}$ as discussed in Fig. 5. Instead, particles transporting through impurity modes do not carry a measurable angular momentum.
[1] Y. Kawano and S. Komiyama, Phys. Rev. B 68, 085328 (2003).

[2] K. L. Grosse, M.-H. Bae, F. Lian, E. Pop, and W. P. King, Nat. Nanotechnol. 6, 287 (2011).

[3] M. Glück, A. R. Kolovsky, and H. J. Korsch, Phys. Rep. 366, 103 (2002).

[4] A. R. Kolovsky and H. J. Korsch, Int. J. Mod. Phys. B 18, 1235 (2004).

[5] G. H. Wannier, Phys. Rev. 117, 432 (1960).

[6] E. E. Mendez, F. Agulló-Rueda, and J. M. Hong, Phys. Rev. Lett. 60, 2426 (1988).

[7] S. R. Wilkinson, C. F. Bharucha, K. W. Madison, Q. Niu, and M. G. Raizen, Phys. Rev. Lett. 76, 4512 (1996).

[8] S. Mukherjee, A. Spracklen, D. Choudhury, N. Goldman, P. Öhberg, E. Andersson, and R. R. Thomson, New J. Phys. 17, 115002 (2015).

[9] C. Schmidt, J. Bühler, A.-C. Heinrich, J. Allerbeck, R. Podzimski, D. Berghoff, T. Meier, W. G. Schmidt, C. Reichl, W. Wegscheider et al., Nat. Commun. 9, 2890 (2018).

[10] W.-R. Lee and K. Park, Phys. Rev. B 89, 205126 (2014).

[11] J. Li, C. Aron, G. Kotliar, and J. E. Han, Phys. Rev. Lett. 114, 226403 (2015).

[12] A. R. Kolovsky, Phys. Rev. A 98, 013603 (2018).

[13] M. Schulz, C. A. Hooley, R. Moessner, and F. Pollmann, Phys. Rev. Lett. 122, 040606 (2019).

[14] E. van Nieuwenburg, Y. Baum, and G. Refael, Proc. Natl. Acad. Sci. USA 116, 9269 (2019).

[15] K. W. Kim, W.-R. Lee, Y. B. Kim, and K. Park, Nat. Commun. 7, 13489 (2016).

[16] K. W. Kim, H. Kwon, and K. Park, Phys. Rev. B 99, 115136 (2019).

[17] W.-R. Lee and K. Park, Phys. Rev. B 92, 195144 (2015).

[18] E. J. Sie, C. H. Lui, Y.-H. Lee, J. Kong, and N. Gedik, Nano Lett. 16, 7421 (2016).

[19] S. Y. Kruchinin, F. Krausz, and V. S. Yakovlev, Rev. Mod. Phys. 90, 021002 (2018).

[20] Y. Jin, L. Gao, J. Chen, C. Wu, J. L. Reno, and S. Kumar, Nat. Commun. 9, 1407 (2018).

[21] L. Bosco, M. Franckié, G. Scalari, M. Beck, A. Wacker, and J. Faist, Appl. Phys. Lett. 115, 010601 (2019).

[22] M. Glück, A. Kolovsky, H. J. Korsch, and N. Moiseyev, Eur. Phys. J. D 4, 239 (1998).
[23] M. Glück, A. R. Kolovsky, and H. J. Korsch, J. Opt. B 2, 694 (2000).

[24] M. Glück, F. Keck, and H. J. Korsch, Phys. Rev. A 66, 043418 (2002).

[25] A. Gómez-León and G. Platero, Phys. Rev. Lett. 110, 200403 (2013).

[26] T. Kitagawa, E. Berg, M. Rudner, and E. Demler, Phys. Rev. B 82, 235114 (2010).

[27] N. H. Lindner, G. Refael, and V. Galitski, Nat. Phys. 7, 490 (2011).

[28] M. S. Rudner, N. H. Lindner, E. Berg, and M. Levin, Phys. Rev. X 3, 031005 (2013).

[29] M. S. Rudner and N. H. Lindner, arXiv:1909.02008 (2019).

[30] M. S. Rudner and N. H. Lindner, arXiv:2003.08252 (2020).

[31] S.-C. Lee and A. Wacker, Phys. Rev. B 66, 245314 (2002).

[32] S.-C. Lee, F. Banit, M. Woerner, and A. Wacker, Phys. Rev. B 73, 245320 (2006).

[33] A. Wacker, M. Lindskog, and D. O. Winge, IEEE J. Sel. Topics Quantum Electron. 19, 1 (2013).

[34] M. Fischetti, J. Appl. Phys. 83, 270 (1998).

[35] S. Rott, N. Linder, and G. H. Döhler, Phys. Rev. B 65, 195301 (2002).

[36] N. Goldman, J. Beugnon, and F. Gerbier, Phys. Rev. Lett. 108, 255303 (2012).

[37] K. C. Nowack, E. M. Spanton, M. Baenninger, M. König, J. R. Kirtley, B. Kalisky, C. Ames, P. Leubner, C. Brüne, H. Buhmann et al., Nat. Mater. 12, 787 (2013).

[38] C.-Z. Chang, J. Zhang, X. Feng, J. Shen, Z. Zhang, M. Guo, K. Li, Y. Ou, P. Wei, L.-L. Wang et al., Science 340, 167 (2013).

[39] M. Aidelsburger, M. Lohse, C. Schweizer, M. Atala, J. T. Barreiro, S. Nascimbène, N. Cooper, I. Bloch, and N. Goldman, Nat. Phys. 11, 162 (2015).

[40] Z. Wu, L. Zhang, W. Sun, X.-T. Xu, B.-Z. Wang, S.-C. Ji, Y. Deng, S. Chen, X.-J. Liu, and J.-W. Pan, Science 354, 83 (2016).

[41] N. R. Cooper, J. Dalibard, and I. B. Spielman, Rev. Mod. Phys. 91, 015005 (2019).

[42] S. Krinner, M. Lebrat, D. Husmann, C. Grenier, J.-P. Brantut, and T. Esslinger, Proc. Natl. Acad. Sci. USA 113, 8144 (2016). 